\title{
Recombinant Vaccinia-Multiepitope Melanoma Peptides-B7.1-B7.2 Vaccine
}

National Cancer Institute

\section{Source}

National Cancer Institute. Recombinant Vaccinia-Multiepitope Melanoma Peptides-B7.1B7.2 Vaccine. NCl Thesaurus. Code C48638.

A cancer vaccine consisting of an inactivated recombinant vaccinia virus encoding epitope peptides derived from melanoma-related HLA-A2-restricted tumor-associated antigens (TAAs), including Melan-A(27-35), gp100(280-288) and tyrosinase(1-9), and two costimulatory B7 proteins, B7.1 (CD80) and B7.2 (CD86). Upon administration, recombinant vaccinia-multiepitope melanoma peptides-B7.1-B7.2 vaccine may stimulate a cytotoxic T-lymphocyte response against melanoma cells that express TAAs which share epitopes with the epitope peptides expressed by the vaccine viral vector, resulting in tumor cell lysis; vaccine viral vector-expressed co-stimulatory proteins B7.1 and B7.2 may enhance the cytotoxic T-lymphocyte immune response to the TAAs. 